\title{
Solvent-dependent phases and phase transformations of a family of 2D halogen-bonded networks
}

\author{
T. M. Roseveare ${ }^{1}$, C. Wilde ${ }^{1}$, V. Csonka ${ }^{1}$ and L. Brammer ${ }^{1}$ \\ ${ }^{I}$ Department of Chemistry, The University of Sheffield, Sheffield, United Kingdom
}

Email: tom.roseveare@sheffield.ac.uk

Molecules can crystallise either in the presence or absence of the solvent used to crystallise them with a range of intermolecular interactions between both the molecule and the solvent occurring to sustain and propagate the crystal structure. Molecules that crystallise as solvates or clathrates could be considered as host-guest materials, but it is often unclear whether a guest-free material can be obtained by heating the solvated material. If the solvent can be removed this can, in turn, lead to vacant void spaces or a partially closed material that can be used to store a secondary guest (either a gas or secondary solvent). Understanding how these materials behave upon removal of the solvent contained within them is crucial in assessing their potential applications. With the CSD recently reaching 1 million deposited crystal structures [1] there is a large resource of untested solvate structures, which may provide inspiration for new guest-uptake materials.[2]

This work presents an attempt to further understand a previously reported family of halogen-functionalised organic molecules which has been reported in 3 distinct phases (two inclusion phases and one solvent-excluded phase)[3]. The two inclusion phases adopt a 2D halogen-bonding network propagated through a halogen-halogen bonded trimer. The work presented here, initially focusing on the bromine-functionalised host molecule, used liquid-assisted grinding to screen a series of solvents to identify desirable inclusion phases. The grinding experiments also identified a previously unreported inclusion phase. Thermal stability studies demonstrated that these inclusion phases transformed to the solvent-excluded phase upon heating. Further work has involved altering the halogen functionality (using fluorine, chlorine or iodine) to see how this affects the propensity to form the desired inclusion phases and the thermal stability of these phases, as well as exploring whether phase transformation can be observed when samples are exposed to a vapour environment.

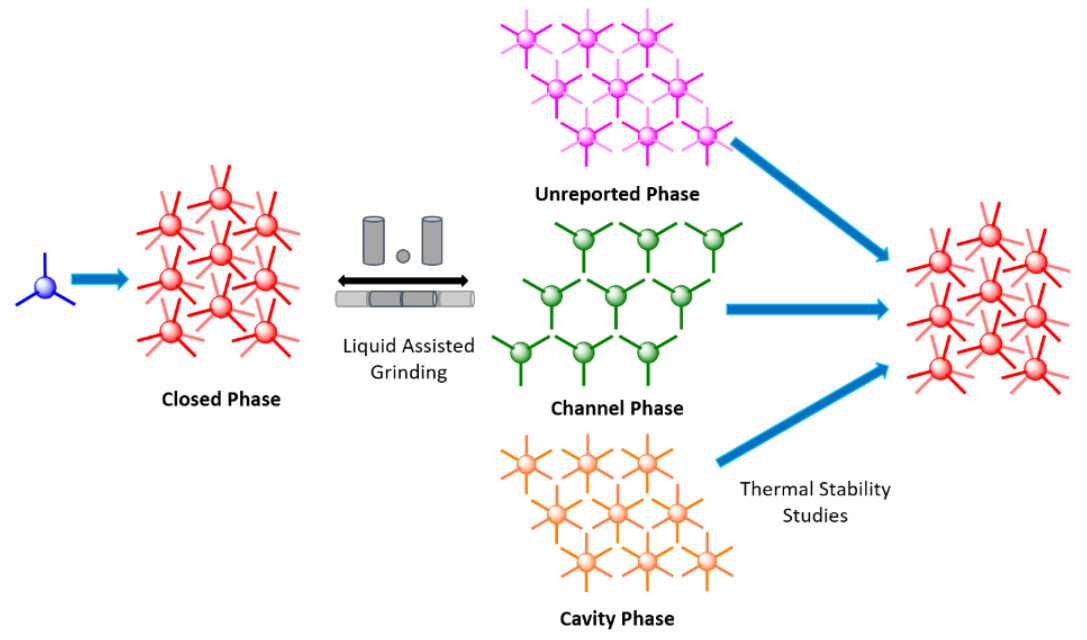

Figure 1. Overview of the solvent-dependent phases of a family of halogen-bonded networks.

[1] R. Taylor, P. A. Wood, Chem. Rev., 2019, 119, 9427-9477.

[2] N.B. McKeown, J. Mater. Chem., 2010, 20, 10588-10597.

[3] B. K. Saha, R. K. R. Jetti, L. S. Reddy, S. Aitipamula and A. Nangia, Cryst. Growth Des., 2005, 5, 887-899.

\section{Keywords: porosity, halogen bonding, liquid-assisted grinding}

The authors $C W$ and VC would like to acknowledge support from the Sheffield Undergraduate Research Experience and the Nuffield Foundation, respectively, for support. 